\title{
Self-dual fields and causality
}

\author{
H. O. Girotti \\ Instituto de Física, Universidade Federal do Rio Grande do Sul, Caixa Postal 15051, 91500 Porto Alegre, \\ Rio Grande do Sul, Brazil
}

\author{
M. Gomes, V. O. Rivelles, and A. J. da Silva \\ Instituto de Física da Universidade de São Paulo, Departamento de Física Matemática, \\ Caixa Postal 20516, 01498 São Paulo, São Paulo, Brazil
}

(Received 9 January 1989)

\begin{abstract}
Causality aspects of two-dimensional self-dual fields are considered. We prove that there is no causal propagation for dimensionless self-dual fields whose Lagrangian does not contain dimensional parameters. It is shown that causal self-dual bosons are possible in the chiral Schwinger model which contains a dimensional charge.
\end{abstract}

The quantization of self-dual fields has aroused some interest, 1,2 particularly due to its relevance to the heterotic string. ${ }^{3}$ More basically, self-dual fields are the building blocks in terms of which the usual fields can be constructed. ${ }^{4}$ However, the quantization of these fundamental objects is beset with notorious difficulties and up to now no covariant Lagrangian describing scalar selfdual fields is known. ${ }^{3}$ More recently, some understanding of the problem has been achieved by Floreanini and Jackiw $^{5}$ who have proposed the following alternatives: (i) a nonlocal Lagrangian in terms of a local field; (ii) a local Lagrangian in terms of a nonlocal field; and (iii) a local Lagrangian in terms of a local field. These alternatives are just different descriptions of the same theory. The formulations (i) and (ii) which exhibit second-class constraints ${ }^{6,7}$ turn out to be invariant under contracted Poincaré transformations, while the fermionic formulation (iii) is manifestly Poincaré invariant. Furthermore, the Becchi-Rouet-Stora-Tyutin (BRST) quantization of Siegel's Lagrangian ${ }^{8}$ has been presented in Ref. 1. It has been claimed that Siegel's model is equivalent to (ii) (Ref. 9).

Besides Poincaré symmetry a consistent quantum field theory must verify further axioms. In this paper we start by showing that not all the proposals in Ref. 5 satisfy the physical requirement of causality. We then argue that the absence of dimensional parameters in the Lagrangian signals the violation of causality for theories involving only dimensionless self-dual fields. This seems to be the case in Siegel's theory. ${ }^{8}$ We conclude this work by using the chiral Schwinger model to exemplify the occurrence of causal dimensionless self-dual bosons in a theory containing a dimensional coupling constant.

The formulation (i) is described by the nonlocal Lagrangian density [unless otherwise stated, from now on $\left.x \equiv\left(x^{0}, x^{1}\right)\right]$

$$
\mathcal{L}^{(\mathrm{i})}(x)=\frac{1}{4} \int d y^{1} \chi(x) \epsilon\left(x^{1}-y^{1}\right) \dot{\chi}(y)-\frac{1}{2} \chi^{2}(x),
$$

where $y$ labels the coordinate pair $\left(x^{0}, y^{1}\right)$. We shall always be using the metric given by $g^{00}=-g^{11}=1, g^{\mu \nu}=0$ if $\mu \neq v$

The solution for the quantum field operator $\chi(x)$ has been found to be $\mathrm{b}^{5}$

$$
\begin{aligned}
\chi(x)=i \int_{0}^{\infty} d k\left(\frac{k}{2 \pi}\right)^{1 / 2} & {\left[e^{i k\left(x^{0}+x^{1}\right)} a^{\dagger}(k)\right.} \\
& \left.-e^{-i k\left(x^{0}+x^{1}\right)} a(k)\right]
\end{aligned}
$$

with $\left[a(k), a^{\dagger}\left(k^{\prime}\right)\right]=\delta\left(k-k^{\prime}\right)$. Then, one readily obtains

$$
[\chi(x), \chi(0)]=i \delta^{\prime}\left(x^{0}+x^{1}\right) .
$$

Since the right-hand side of (3) is nonvanishing only in the light-cone branch defined by $x^{0}+x^{1}=0$, the quantum theory arising from (1) is compatible with causality. From (3), notice that the dimension of $\chi$, and therefore its spin, is one.

The formulation (ii) is described by the local Lagrangian density

$$
\mathcal{L}_{l}^{(\mathrm{ii})}=\frac{1}{2}\left(\partial_{0} \phi_{l}\right)\left(\partial_{1} \phi_{l}\right)-\frac{1}{2}\left(\partial_{1} \phi_{l}\right)\left(\partial_{1} \phi_{l}\right),
$$

where the subscript $l$ (left) indicates that $\phi_{l}$ is a self-dual field obeying the equation $\left(\partial_{0}-\partial_{1}\right) \phi_{l}=0$. The canonical quantization of (4) is straightforward ${ }^{6,7}$ and one finds that

$$
\begin{aligned}
& \left\langle 0\left|\phi_{l}(x) \phi_{l}(0)\right| 0\right\rangle \\
& \quad=\frac{1}{2 \pi} \int_{0}^{\infty} \frac{d k}{k}\left[e^{-i k\left(x^{0}+x^{1}\right)}-\theta\left(\mu e^{-e}-k\right)\right] \\
& \quad=-\frac{i}{4} \epsilon\left(x^{0}+x^{1}\right)-\frac{1}{2 \pi} \ln \left(\left|x^{0}+x^{1}\right| \mu\right),
\end{aligned}
$$

where $\mathcal{C}$ is the Euler constant and $\mu$ is an infrared regulator with mass dimension. Although the Lagrangian (4) does not contain dimensional parameters, the theory only acquires a well-defined meaning after the introduction of a dimensional infrared regulator. However, it follows from (5) that the full commutator

$$
\left[\phi_{l}(x), \phi_{l}(0)\right]=-\frac{i}{2} \epsilon\left(x^{0}+x^{1}\right)
$$


is entirely independent of $\mu$. Moreover, the commutator (6) does not vanish outside the light cone. Therefore, the quantum field $\phi_{l}$ is not a causal field. As a consequence, the chronologically ordered product $T\left[\phi_{l}(x) \phi_{l}(0)\right]$ is not a Lorentz-invariant operator. Notice that, this time, the basic commutator (6) is dimensionless, in agreement with the fact that $\phi_{1}$ is a dimensionless field.

One arrives at a similar conclusion for the $r$ (right) field:

$$
\mathcal{L}_{r}^{(\mathrm{ii})}=\frac{1}{2}\left(\partial_{0} \phi_{r}\right)\left(\partial_{1} \phi_{r}\right)+\frac{1}{2}\left(\partial_{1} \phi_{r}\right)\left(\partial_{1} \phi_{r}\right)
$$

obeying $\left(\partial_{0}+\partial_{1}\right) \phi_{r}=0$. Indeed,

$$
\left[\phi_{r}(x), \phi_{r}(0)\right]=-\frac{i}{2} \epsilon\left(x^{0}-x^{1}\right) .
$$
field

In spite of these causality problems, the ordinary scalar

$$
\phi(x)=\phi_{l}(x)+\phi_{r}(x),
$$

with $\left[\phi_{l}(x), \phi_{r}(0)\right]=0$, is causal. In fact, it satisfies

$$
[\phi(x), \phi(0)]=-i \theta\left(x^{2}\right) \epsilon\left(x^{0}\right)
$$

which, of course, vanishes outside the light cone $\left(x^{2}<0\right)$.

The formulation (iii),

$$
\mathcal{L}^{(\mathrm{iii})}=i u^{\dagger}\left(\partial_{0} u-\partial_{1} u\right),
$$

describes a Weyl fermion obeying $\left(\partial_{0}-\partial_{1}\right) u=0$. The canonical quantization of (11) leads to

$$
\begin{aligned}
& u(x)=\frac{1}{\sqrt{2 \pi}} \int_{0}^{\infty} d x\left[e^{i k\left(x^{0}+x^{1}\right)} b^{\dagger}(k)\right. \\
& \left.+e^{-i k\left(x^{0}+x^{1}\right)} a(k)\right], \\
& u^{\dagger}(x)=\frac{1}{\sqrt{2 \pi}} \int_{0}^{\infty} d x\left[e^{i k\left(x^{0}+x^{1}\right)} a^{\dagger}(k)\right. \\
& \left.+e^{-i k\left(x^{0}+x^{1}\right)} b(k)\right],
\end{aligned}
$$

where

$$
\left\{a(k), a^{\dagger}\left(k^{\prime}\right)\right\}=\left\{b(k), b^{\dagger}\left(k^{\prime}\right)\right\}=\delta\left(k-k^{\prime}\right),
$$

while all other anticommutators vanish. One then obtains

$$
\begin{aligned}
& \{u(x), u(0)\}=\left\{u^{\dagger}(x), u^{\dagger}(0)\right\}=0, \\
& \left\{u(x), u^{\dagger}(0)\right\}=\delta\left(x^{0}+x^{1}\right),
\end{aligned}
$$

which are all compatible with causality. To the same results one arrives using the bosonization formulas ${ }^{5,7}$

$$
\begin{aligned}
& u(x)=\left[\frac{\mu}{2 \pi}\right]^{1 / 2}: \exp \left[-i(2 \pi)^{1 / 2} \phi_{l}(x)\right]:, \\
& u^{\dagger}(x)=\left(\frac{\mu}{2 \pi}\right)^{1 / 2}: \exp \left[i(2 \pi)^{1 / 2} \phi_{l}(x)\right]: .
\end{aligned}
$$

In Ref. 7 a whole class of self-dual soliton fields depending on a real parameter was introduced. They are described by the fields

$$
u_{\gamma}(x)=\left(\frac{\mu}{2 \pi}\right)^{\gamma^{2} / 4 \pi}: \exp \left[-i \gamma \phi_{l}(x)\right]:
$$

which have dimension $=\operatorname{spin}=\gamma^{2} / 4 \pi$. For general values of the spin such fields have nonlocal field-dependent (anti)commutation relations. Nevertheless, if the spin is either a nonzero integer or half-integer, the corresponding field is local and satisfies

$$
\begin{aligned}
& {\left[u_{\gamma}(x), u_{\gamma}(0)\right]_{ \pm}=\left[u_{\gamma}^{\dagger}(x), u_{\gamma}^{\dagger}(0)\right]_{ \pm}=0,} \\
& {\left[u_{\gamma}(x), u_{\gamma}^{\dagger}(0)\right]_{ \pm}=2 \pi i \frac{(-1)^{\gamma^{2} / 2 \pi-1}}{\left(\gamma^{2} / 2 \pi-1\right) !} \delta^{\left(\gamma^{2} / 2 \pi-1\right)}\left(x^{0}+x^{1}\right),}
\end{aligned}
$$

where the subscript \pm indicates either commutator or anticommutator. For spin $\frac{1}{2}$ and 1 we reobtain the previously written (anti)commutation relations.

The examples above support the conclusion that the theory of a single dimensionless self-dual field $\varphi_{l}$ necessarily violates causality. In fact, since' the fields only depend on $x$ through the combination $x^{0}+x^{1}$, translation invariance dictates that the vacuum expectation value of the field commutator (or anticommutator), $\langle 0|\left[\varphi_{l}(x)\right.$, $\left.\varphi_{l}(y)\right]|0\rangle$, must be of the form $f\left(x^{0}-y^{0}+x^{1}\right.$ $\left.-y^{1}\right)$, where $f$ is some function. Thus, for $f$ to vanish outside the light cone it must be of the form

$f\left(x^{0}-y^{0}+x^{1}-y^{1}\right)=\mathcal{P}\left(\partial_{1}^{x}\right) \delta\left(x^{0}-y^{0}+x^{1}-y^{1}\right)$,

where $\mathcal{P}$ is some polynomial. Since, by assumption the Lagrangian does not contain dimensional parameters which might compensate for the dimensions of the righthand side of (22), the field commutators cannot be of the form (22) and, as a consequence, causality is violated (we recall that the infrared regulator does not enter in the commutation relation). This is exactly the case of Siegel's theory ${ }^{8}\left(\partial_{ \pm}=\partial / \partial x^{ \pm}, \sqrt{2} x^{ \pm}=x^{0} \pm x^{1}\right)$ :

$$
\mathcal{L}_{S}=\frac{1}{2}\left(\partial_{-} \phi\right)\left(\partial_{+} \phi\right)-\frac{\lambda}{2}\left(\partial_{-} \phi\right)^{2}
$$

in the gauge $\lambda=0$.

We conclude this work by exemplifying the occurrence of a dimensionless self-dual field in a theory containing a dimensional coupling constant. What we have in mind is the gauge-noninvariant version of the chiral Schwinger model, which describes the dynamics of fermions chirally coupled to a vector field $A^{\mu}$ in a $(1+1)$-dimensional space-time. After bosonization the effective Lagrangian $\mathcal{L}$ turns out to be $\mathcal{L}=\mathcal{L}\left(A^{\mu}, \phi, e, a\right)$ where $\phi$ is the bosonizing field, $e$ is a dimensional coupling constant, and $a$ is a real parameter reflecting the ambiguity in the computation of the fermionic determinant. ${ }^{10}$ The only physically meaningful regions are $a>1$ and $a=1$. The theory has been canonically quantized in both of these regions. ${ }^{11}$ In particular, for $a=1$ one finds that $\phi$ is a free massless scalar field and that $A^{\mu}$ is a dimensionless self-dual field given by ${ }^{11}\left(\widetilde{\partial}^{\mu}=\epsilon^{\mu \nu} \partial_{v}, \epsilon^{\mu \nu}=-\epsilon^{v \mu}, \epsilon^{01}=1\right)$

$$
A^{\mu}=-\frac{1}{e}\left(\partial^{\mu}+\widetilde{\partial}^{\mu}\right) \phi
$$

which in turn implies that 


$$
A^{1}(x)=-A^{0}(x)=\frac{\sqrt{2}}{e} \partial_{+} \phi .
$$

From (10) and (25) one finds

$$
\left[A^{1}(x), A^{1}(0)\right]=\frac{2 i}{e^{2}} \delta^{\prime}\left(x^{0}+x^{1}\right)
$$

in agreement with causality. Notice that the presence of $e^{-2}$ makes the right-hand side of (26) dimensionless.

We are indebted to Professor R. Jackiw for a critical reading of the manuscript and many valuable comments. This work was partially supported by Conselho Nacional de Desenvolvimento Científico e Tecnológico Brazil).
${ }^{1}$ J. M. F. Labastida and M. Pernici, Nucl. Phys. B297, 557 (1988); C. Imbimbo and A. Schwimmer, Phys. Lett. B 193, 435 (1987).

${ }^{2}$ L. Mezincescu and R. I. Nepomechie, Phys. Rev. D 37, 3067 (1988); J. Sonnenschein, Nucl. Phys. B309, 752 (1988); M. Gomes, V. O. Rivelles, and A. J. da Silva, Phys Lett. B 218, 63 (1989).

${ }^{3}$ J. M. F. Labastida and M. Pernici, Phys. Rev. Lett. 59, 2511 (1987).

${ }^{4}$ M. Gomes, V. Kurak, V. O. Rivelles, and A. J. da Silva, Phys. Rev. D 38, 1344 (1988).
${ }^{5}$ R. Floreanini and R. Jackiw, Phys. Rev. Lett. 59, 1873 (1987).

${ }^{6}$ M. E. V. Costa and H. Girotti, Phys. Rev. Lett. 60, 1771 (1988).

${ }^{7}$ H. O. Girotti, M. Gomes, V. Kurak, V. O. Rivelles, and A. J. da Silva, Phys. Rev. Lett. 60, 1913 (1988).

${ }^{8}$ W. Siegel, Nucl. Phys. B238, 307 (1984).

${ }^{9}$ J. Sonnenschein, Phys. Rev. Lett. 60, 1772 (1988).

${ }^{10}$ R. Jackiw and R. Rajaraman, Phys. Rev. Lett. 54, 1219 (1985).

${ }^{11}$ H. O. Girotti, K. D. Rothe, and H. J. Rothe, Phys. Rev. D 33, 514 (1986); 34, 592 (1986); H. O. Girotti and K. D. Rothe, Int. J. Mod. Phys. (to be published). 